\title{
Lattice gas cellular automata model for rippling and aggregation in myxobacteria
}

\author{
Mark S. Alber ${ }^{\mathrm{a}}$, Yi Jiang ${ }^{\mathrm{b}}$ and Maria A. Kiskowski ${ }^{\mathrm{a}}$ \\ ${ }^{a}$ Department of Mathematics and the Interdisciplinary Center for the Study of \\ Biocomplexity, University of Notre Dame, Notre Dame, IN 46556-5670 \\ b Theoretical Division, Los Alamos National Laboratory, Los Alamos, NM 87545
}

\begin{abstract}
A lattice-gas cellular automaton (LGCA) model is used to simulate rippling and aggregation in myxobacteria. An efficient way of representing cells of different cell size, shape and orientation is presented that may be easily extended to model later stages of fruiting body formation. This LGCA model is designed to investigate whether a refractory period, a minimum response time, a maximum oscillation period and non-linear dependence of reversals of cells on C-factor are necessary assumptions for rippling. It is shown that a refractory period of 2-3 minutes, a minimum response time of up to 1 minute and no maximum oscillation period best reproduce rippling in the experiments of Myxoccoccus xanthus. Non-linear dependence of reversals on C-factor is critical at high cell density. Quantitative simulations demonstrate that the increase in wavelength of ripples when a culture is diluted with non-signaling cells can be explained entirely by the decreased density of C-signaling cells. This result further supports the hypothesis that levels of C-signaling quantitatively depend on and modulate cell density. Analysis of the interpenetrating high density waves shows the presence of a phase shift analogous to the phase shift of interpenetrating solitons. Finally, a model for swarming, aggregation and early fruiting body formation is presented.
\end{abstract}

Key words: pattern formation, cellular automata, aggregation, statistical mechanics, myxobacteria, rippling, collective behavior PACS: 87.18.Bb, 87.18.Ed, 87.18.Hf, 87.18.La

Email addresses: malber@nd.edu (Mark S. Alber), jiang@lanl.gov (Yi Jiang), mkiskows@nd.edu (Maria A. Kiskowski). 


\section{Introduction}

Myxobacteria are one of the prime model systems for studying cell-cell interaction and cell organization preceding differentiation. Myxobacteria are social bacteria which swarm, feed and develop cooperatively [1]. When starved, myxobacteria self-organize into a three-dimensional fruiting body structure. Fruiting body formation is a complex multi-step process of alignment, rippling, streaming and aggregation that culminates in the differentiation of highly elongated, motile cells into round, non-motile spores. A successful model exists for the fruiting body formation of the eukaryotic slime mold Dictyostelium discoideum $[2,3,4]$. Understanding the formation of fruiting bodies in myxobacteria, however, would provide a new insight since collective myxobacteria motion depends not on chemotaxis as in Dictyostelium but on contact-mediated signaling (see [5] for a review).

During fruiting body formation myxobacteria cells are elongated, with a 10:1 length to width ratio, and move along surfaces by gliding. Gliding occurs in the direction of a cell's long axis [6] and is controlled by two distinct motility systems in myxobacteria $[7,8]$. One of the most interesting patterns that develops during myxobacteria morphogenesis is rippling, which often occurs spontaneously and transiently during the aggregation phase $[9,10,11]$. Rippling myxobacteria form equidistant ridges of high cell density which appear to advance through the population as rhythmically traveling waves $[9,10]$ (Figure 1). Cell movement in a ripple is approximately one-dimensional since the majority of cells are aligned and move in parallel lines with or against the direction of wave propagation [12]. Tracking individual bacteria within a ripple has shown that cells reverse their traveling directions back and forth and that each travels on the order of one wavelength between reversals [12]. The ripple waves propagate with no net transport of cells [12] and wave overlap causes neither constructive nor destructive interference [12]. Although mechanisms for gliding are not fully understood, they are believed to account for both alignment [7,13] and reversals [7] in myxobacteria.

Rippling is related to a membrane-associated signaling protein called $C$-factor. C-factor regulates rippling $[10,12,14]$, cells without the ability to produce Cfactor fail to ripple [10] and the addition of C-factor (extracted from fruiting body cultures) causes cell reversal frequencies to increase three-fold [12]. Csignaling occurs via the direct cell-cell transfer of C-factor when two elongated cells collide head to head $[12,15,16,17,18]$. Understanding the mechanisms of the rippling phase may reveal many clues about the way myxobacteria organize collective motion since C-factor is also involved in all other stages of fruiting body formation. For example, cells lacking in C-factor fail to aggregate or sporulate $[19,20,21]$ while high concentrations of exogeneous C-factor induce aggregation and sporulation [16,20,22,23]. 


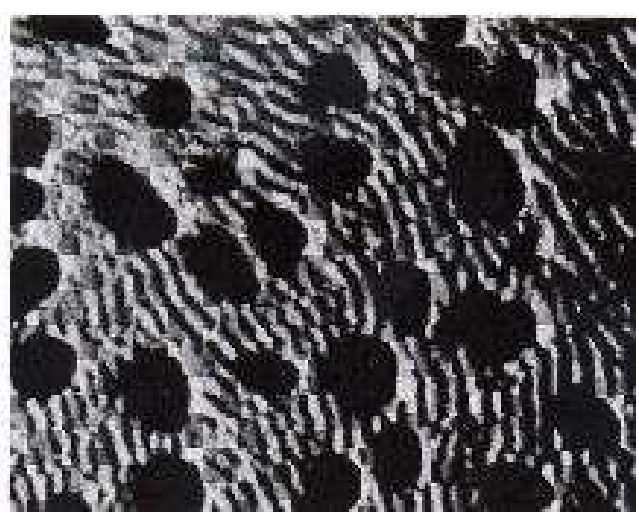

Fig. 1. A field of immature fruiting bodies, shown as dark patches, with ripples formed by cells outside of the aggregates. (From Shimkets and Kaiser [10] with permission.)

In this paper we use two lattice gas cellular automata (LGCA) models to simulate rippling and aggregation during the fruiting body formation of myxobacteria, to show the potential of cellular automata as models for biological pattern formation processes, and to evaluate, in particular, the necessity of different biological assumptions shown in previous models for pattern formation in myxobacteria.

Sager and Kaiser [12] have proposed that precise reflection explains the lack of interference between wave-fronts for myxobacteria rippling. Oriented collisions between cells initiate C-signaling that causes cell reversals. According to this hypothesis of precise reflection, when two wave-fronts collide, the cells reflect one another, pair by pair, in a precise way that preserves the wave structure in mirror image. Figure 2 shows a schematic diagram of this reflection.

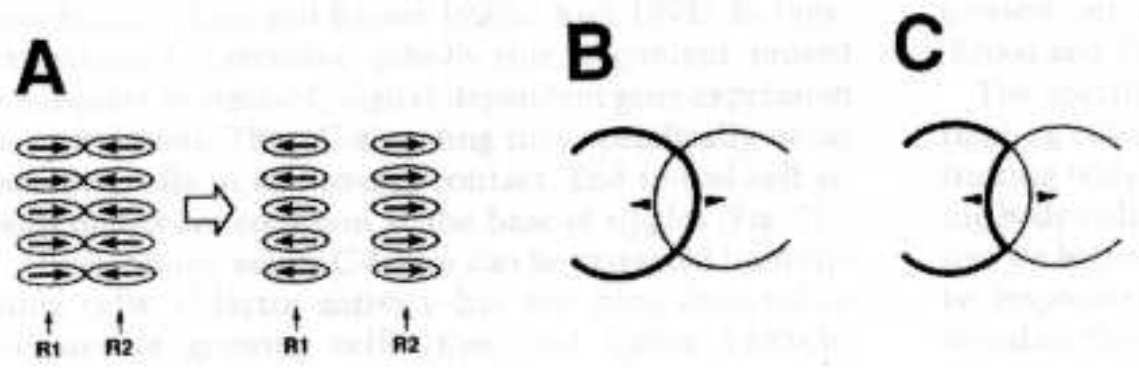

Fig. 2. (A) A reflection model for the interaction between individual cells in two counter-migrating ripple waves. Laterally aligned cells in counter-migrating ripples (labeled R1 and R2) reverse upon end to end contact. Arrows represent the directions of cell movement. Relative cell positions are preserved. (B) Morphology of ripple waves after collision. Thick and thin lines represent rightward and leftward moving wave fronts, respectively. Arrows show direction of wave movement. (C) Reflection of the same waves shown in $\mathrm{B}$, with the ripple cell lineages modified to illustrate the effect of reversal. (From Sager and Kaiser [12] with permission). 
We present a new LGCA approach for modeling cells which is computationally efficient yet approximates continuum dynamics more closely than assuming point-like cells. As an example of this new approach, we present a model for myxobacteria rippling based on the hypothesis of precise reflection and a model for aggregation based on C-signaling.

This paper is organized as follows. The biological assumptions for precise reflection and $\mathrm{C}$-signaling that motivate the models are described in the next section. In section 3 we describe specifics of two LGCA models. In section 4 results of modeling rippling phenomenon are discussed in detail. Section 5 provides description of a model for aggregation centers. The paper ends with a summary section.

\section{Biological Background}

In this section we describe the biological observations which motivate our models for rippling and aggregation.

Rippling and aggregation are both controlled by C-signaling and are characterized by specific high cell density patterns (in particular, moving high density ridges in rippling and stationary high density mounds in aggregation). There is a marked relationship between cell density, levels of C-signaling and behaviors in myxobacteria triggered by C-signaling [24]. C-signaling increases with density since end-to-end contacts between cells are more likely with increased density $[25,26]$ and high cell densities favor spatial arrangements in which there are many end-to-end contacts due to the polarity of myxobacterial cells $[25,26]$. Cell density and C-signaling levels increase together from rippling to aggregation and from aggregation to sporulation $[25,26]$. Further, increased thresholds of C-factor induce rippling, aggregation and sporulation respectively [22,23,27], suggesting C-signaling levels, as a measure of cell density, are checkpoints for different stages of development. Kim and Kaiser suggest that C-factor may act as a developmental timer that triggers sporulation only when cell density is as high as possible [17,22]. A high density aggregate will culminate in a fruiting body with a large number of spores ensuring that the next cycle is started by a population of cells [28]. Sager and Kaiser have also observed the effect of C-signaling-competent cell density upon ripple wavelength [12]. They dilute a cell population of C-signaling-competent cells with cells that are able to respond to C-factor but are not able to transmit it. They find that with increased concentrations of these csgA-minus cells, ripple wavelength increases non-linearly.

In addition to cell density patterns, cell geometries are important throughout the stages of fruiting body formation and distinguish different stages. Dur- 
ing the fruiting body formation, cells form aligned patches from a random distribution [29]. For rippling, a large number of cells must be aligned both parallel and anti-parallel within the same field. For streaming, cells form long chains which flow cooperatively in aggregation centers [30]. In Stigmatella spp., cells moving in circles or spirals form microscopic transient aggregates. These aggregates disappear as cells also spiral away tangentially [31]. Macroscopic aggregates form in areas of high density [31] and may also disappear as cells apparently stream along chains from one aggregate to the other [32]. The mature structures of fruiting bodies are diverse and species-dependent, ranging in size between 10 and $1000 \mu \mathrm{m}$ [28]. In Myxococcus xanthus, the basal region of the fruiting body is a shell of densely packed cells which orbit in two directions, both clock-wise and counter-clockwise, around an inner region only one-third as dense [25,26]. In Stigmatella aggregates, cells are organized in concentric circles or ellipses and cells move in a spiral fashion up the aggregate as the fruiting body develops [31,33].

Current models for rippling $([34,35,36])$ assume precise reflection. Key differences among these models include their biological assumptions regarding the existence of internal biochemical cell cycles. It is still not known if an internal cell timer is involved in myxobacterial rippling. Several models with completely different assumptions all qualitatively produce ripple patterns resembling experiment.

An internal timer is a hypothetical molecular cell clock which regulates the interval between reversals. The internal timer may specify a delay, or minimum period between reversals, which would include the refractory period, see below, and a minimum response time; the minimum period of time required for a non-refractory cell to become stimulated to turn. Also, the internal timer may specify a maximum oscillation period, in which case the timer may speed up or slow down depending upon collisions, but the cell will always turn within a specified period of time even without collisions. Individual pre-rippling cells reverse spontaneously every 5-10 minutes with a variance in the period much smaller than the mean $[35,37,38]$. This would suggest that there is a component of the timer specifying a maximum oscillation period. Also, observation of rippling bacteria reveals that cells oscillate even in ripple troughs where the density is too low for frequent collisions [12] further supporting the hypothesis of a maximum oscillation period.

The refractory period is a period of time immediately following a cell reversal, during which the cell is insensitive to C-factor. Although there is no evidence of a refractory period in the C-signaling system, the refractory period is a general feature of bacterial signaling systems [35] (for a description of the role the refractory period plays in Dictyostelium, see [39]). The addition of .02 units of external C-factor triples the reversal frequency of single cells from .09 reversals per minute to .32 reversals per minute [12]. Cells do not reverse more 
frequently at still higher levels of C-factor, however, suggesting the existence of a minimum oscillation period of 3 minutes in response to C-factor. This minimum oscillation period would be the sum of the refractory period and the minimum response, so the duration of the refractory period cannot be guessed from this fact alone.

To resolve the conflicts of these models for rippling our first LGCA model is designed to test different assumptions. The results of our model for rippling shows that rippling is stable for a wide range of parameters, C-signaling plays an important role in modulating cell density during rippling, and non-Csignaling cells have no effect on the rippling pattern when mixed with wild-type cells. Further, by comparing model results with experiments, we can conclude reversals during rippling would not be regulated by a built-in maximum oscillation period.

We then present a second LGCA model for aggregation based on C-signal alignment, which reproduces the sequence and geometry of the non-rippling stages of fruiting body formation in detail, showing that a simple local rule based on C-signaling can account for many experimental observations.

\section{Model and Method}

LGCA are relatively simple Cellular Automata models. They employ a regular, finite lattice and include a finite set of particle states, an interaction neighborhood and local rules that determine the particles' movements and transitions between states [40]. LGCA differ from traditional CA by assuming particle motion and an exclusion principle. The connectivity of the lattice fixes the number of allowed non-zero velocities or channels for each particle. For example, a nearest-neighbor square lattice has four non-zero allowed channels. The channel specifies the direction and magnitude of movement, which may include zero velocity (resting). In a simple exclusion rule, only one particle may have each allowed non-zero velocity at each lattice site. Thus, a set of Boolean variables describes the occupation of each allowed particle state: occupied (1) or empty (0). Each lattice site on a square lattice can then contain from zero to four particles with non-zero velocity.

The transition rule of an LGCA has two steps. An interaction step updates the state of each particle at each lattice site. Particles may change velocity state, appear or disappear in any number of ways as long as they do not violate the exclusion principle. In the transport step, cells move synchronously in the direction and by the distance specified by their velocity state. Synchronous transport prevents particle collisions which would violate the exclusion principle (other models define a collision resolution algorithm). LGCA models are 
specially constructed to allow parallel synchronous movement and updating of a large number of particles [40].

\subsection{Representation of cells}

In classical LGCA, biological cells are dimensionless and represented as a single occupied node on a lattice (e.g., see [34] and [36]). Interaction neighborhoods are typically nearest-neighbor or next-nearest-neighbor on a square lattice. The exclusion principle makes transport unwieldy when a single cell occupies more than one node since a cell may only advance if all the channels it would occupy are available. Similarly, it is difficult to model the overlapping and stacking of cells. Cells without dimension are untenable for a sophisticated model of myxobacteria fruiting body formation, however. Cells are very elongated during rippling, streaming and aggregation and form regular, dense arrays by cell alignment. Also, a realistic model of cell overlap and cell stacking is needed since interaction occurs only at specific regions of highly elongated cells and cell density is a critical parameter throughout this morphogenesis.

Börner et al. [34] have mediated the problem of stacking by introducing a semithree-dimensional lattice where a third z-coordinate gives the vertical position of each cell when it is stacked upon other cells. Stevens [41] has introduced a model of rod-shaped cells that occupy many nodes and have variable shape in her cellular automata model of streaming and aggregation in myxobacteria. Neither of these two models are LGCA since they do not incorporate synchronous transport along channels. We device a novel way of representing cells which facilitates variable cell shape, cell stacking and incomplete cell overlap while preserving the advantages of LGCA; namely, synchronous transport and binary representation of cells within channels (e.g., a ' 0 ' indicating an unoccupied channel and a ' 1 ' indicating an occupied channel).

We represent the cells as (1) a single node which corresponds to the position of the cell's center (or "center of mass") in the $x y$ plane, $(2)$ the choice of occupied channel at the cell's position designating the cell's orientation and (3) a local neighborhood defining the physical size and shape of the cell with associated interaction neighborhoods (Figure 3). The interaction neighborhoods depend on the dynamics of the model and need not exactly overlap the cell shape. In our models for rippling and aggregation, we define the size and shape of the cell as a $3 \times \ell$ rectangle, where $\ell$ is cell length. As $\ell$ increases, the cell shape becomes more elongated. A cell length of $\ell=30$ corresponds to the $1 \times 10$ proportions of rippling Myxococcus xanthus cells [17]. Representing a cell as an oriented point with an associated cell shape is computationally efficient, yet approximates continuum dynamics more closely than assuming point-like cells, since elongated cells may overlap in many ways. We have also solved the 
cell stacking problem, since overlapping cell shapes correspond to cells stacked on top of each other. This cell representation conveniently extends to changing cell dimensions and the more complex interactions of fruiting body formation.

\subsection{LGCA model for rippling}

We assume precise reflection and investigates the roles of a cell refractory period, a minimum response period, a maximum oscillation period and nonlinear dependence of reversals on C-factor independently.

\subsubsection{Local Rules}

(1) Our model employs a square lattice with periodic boundary conditions imposed at all four edges. Unit velocities are allowed in the positive and negative $x$ directions. (A resting channel may be easily added to model a small percentage of resting cells as in [34].

(2) Cells are initially randomly distributed with density $\delta$, where $\delta$ is the total cell area divided by total lattice area.

(3) Every cell is initially equipped with an internal timer by randomly assigning it a clock value between 1 and a maximum clock value $\tau$. We define a refractory period $R$ such that $0 \leq R<\tau$ (see a detailed description of the internal timer, below). If the internal timer $\phi$ of a cell is less than $R$, the cell is refractory. Otherwise, the cell is sensitive.

(4) At each time-step, the internal timer of each refractory cell is increased by 1 while the internal timer of sensitive cells is increased by an amount proportional to the number of head-on cell-cell collisions $n$ occurring at that timestep.

(5) When a cell's internal timer has increased past $\tau$, the cell reverses, the internal timer resets to 0 , and the cell becomes refractory. Reversals occur as a cell's center switches from a right- or left-directed channel to a leftor right-directed channel, respectively.

(6) During the final transport step, all cells move synchronously one node in the direction of their velocity by updating the positions of their centers. Separate velocity states at each node ensure that more than one cell never occupies a single channel.

\subsubsection{Internal timer}

We model an internal timer with three parameters; $R, t$ and $\tau$. $R$ is the number of refractory time-steps, $t$ is the minimum number of time-steps until a reversal and $\tau$ is the maximum number of time-steps until a reversal. The

minimum period of time required for a sensitive cell to become stimulated to 
turn is the minimum response period $t-R$. During the refractory period, cells are insensitive to collisions and the internal timer advances at a uniform rate. After the refractory period, cells become sensitive and during this phase the number of head-on cell-cell collisions accelerates the internal timer so that the interval between reversals shortens. This acceleration is density-dependent, so that many simultaneous collisions accelerate the internal timer more than only one collision.

Our internal timer extends the timer in Igoshin et al. [35]. They used a phase variable $\phi$ to model an oscillating cycle of movement in one direction followed by a reversal and movement in the opposite direction. During the refractory period the phase variable advances at a constant rate but during the sensitive period, the phase variable advance may increase non-linearly with the number of collisions. Thus, the evolution of our timer determines reversal rather than a collision as in the model of Börner et al. [34]. The state of our internal timer is specified by $0 \leq \phi(t) \leq \tau$. $\phi$ progresses at a fixed rate of one unit per time-step for $R$ refractory time-steps, and then progresses at a rate $\omega$ that depends non-linearly on the number of collisions $n$ which have occurred at that timestep to the power $p$ :

$$
\omega(x, \phi, n, q)=1+\left(\frac{\tau-t}{t-R}\right) *\left(\frac{[\min (n, q)]^{p}}{q^{p}}\right) * F(\phi)
$$

where,

$$
F(\phi)= \begin{cases}0, & \text { for } 0 \leq \phi \leq R \\ 0, & \text { for } \pi \leq \phi \leq(\pi+R) \\ 1, & \text { otherwise }\end{cases}
$$

This equation is the simplest that produces a reversal period of $\tau$ when no collisions occur, a refractory period of $R$ time-steps in which the phase velocity is one, and a minimum reversal period of $t$ when a threshold (quorum) number $q$ of collisions occurs at every sensitive time-step. There is "quorum sensing" in that the clock velocity is maximal whenever the number of collisions at a time-step exceeds the quorum value $q$. A particle will oscillate with the minimum reversal period only if it reaches a threshold number of collisions during each non-refractory time-step (for $(t-R)$ time-steps). If the collision rate is below the threshold, the clock phase velocity is less than maximal. However, as the number of collisions increases from 0 to $q$, the phase velocity increases non-linearly as $q$ to the power $p$.

While in the model of Börner et al. [34] there is no minimum response period for a cell to reverse, and in the model of Igoshin et al. [35] a minimum response 
time is an inherent component of the internal clock, our model incorporates "on-off switches" for a refractory period, minimum response period, maximum oscillation period and quorum sensing. Setting the refractory period equal to 0 time-steps in our model is the off-switch for the refractory period, and setting $t=R+1$ is the off-switch for the minimum response time. No maximum oscillation period is modeled by choosing a maximum oscillation period $\tau$ greater than the running period of the simulation, so that the automatic reversal of cells within $\tau$ time-steps has no effect on the dynamics of the simulation. There is no quorum sensing if $q$ is set to 1 so that a single collision during a timestep has the same effect as many collisions.

If there is no refractory period, cells are always sensitive to collisions. If there is no minimum response time, cells may reverse immediately after becoming sensitive if there are sufficiently many collisions in one timestep. Finally, if there is no maximum oscillation period, cells may never reverse without sufficiently many collisions.

\subsubsection{Head-on cell-cell collisions}

We define an interaction neighborhood of eight nodes for the exchange of Cfactor at the poles of a cell of length 1 (see Figure 3). The cell width of 3 nodes is larger than 1 to account for coupling in the y-direction and the interaction neighborhood must extend at least two nodes along the length of the cell to compensate for the discretization of the lattice since cells traveling in opposite directions may pass without their poles exactly overlapping.

A head-on cell-cell collision is defined to occur when the interaction neighborhoods of two anti-parallel cells overlap. A cell may collide with multiple cells simultaneously since the interaction neighborhood is four nodes at each pole. Note that the specific shape of the cell is not important for rippling dynamics since the two areas of C-signaling are the only places where interaction occurs. Nevertheless, a shape extending over several nodes is necessary to permit the necessary overlapping and stacking at high density since the exclusion principle mandates that each channel has at most one cell center. Thus, the cell centers of two colliding cells will be separated by one cell length and do not compete for channels at the same node. Also, for sufficiently long cell lengths, the probability of more than one cell center located at the same node is low even when the local cell density may be high.

We are able to simulate a rippling population with arbitrary concentrations of both wild-type and non-C-signaling cells and quantitatively reproduce their experimental results in detail, as did Igoshin et al. using their continuum model [35]. Further, we demonstrate that the change in wavelength may be entirely explained by the change in density of $\mathrm{C}$-signaling cells. 


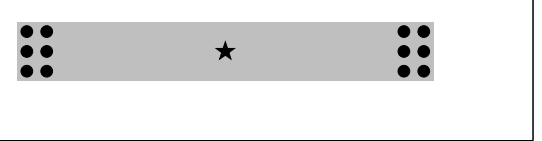

Fig. 3. The shaded rectangle corresponds to the cell shape of a right or left moving cell in our model for rippling. This cell is $3 \times 21$ nodes for a $1 \times 7$ aspect ratio. The star in this figure corresponds to the cell's center and the nodes of the interaction neighborhood where C-factor is exchanged are indicated by black squares at the cell poles.

\section{Rippling results and discussion}

Our model forms a stable ripple pattern from a homogeneous initial distribution for a wide range of parameters, with the ripples apparently differing only in ripple wavelength, ripple density and ripple width (see Figure 4).

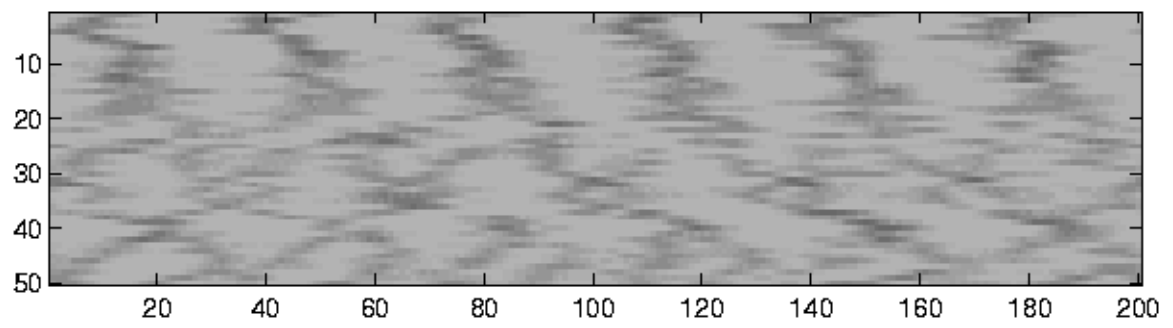

Fig. 4. Typical ripple pattern including both a cell clock and refractory period in the model. (Cell length $=5, \delta=2, R=10, t=15, \tau=25$.) Figure shows the density of cells (darker gray indicates higher density) on a $50 \times 200$ lattice after 1000 time steps, corresponding to approximately 200 minutes in real time.

Absence of a maximum oscillation period is modeled by choosing a maximum oscillation period $\tau$ greater than the running period of the simulation, so that the automatic reversal of cells within $\tau$ time-steps has no effect on the dynamics of the simulation. We find that ripples form with or without a maximum oscillation period over the full range of densities. When there is a maximum oscillation period, the maximum oscillation period must be chosen greater than twice the refractory period for the development of ripples. There is no upper bound on the maximum oscillation time, which is why the maximum reversal period is unnecessary. Ripples develop most quickly and cell oscillations are most regular with an internal timer when the maximum oscillation period is carefully chosen with respect to the other parameters of the model. Nevertheless, it appears that experimental results are best reproduced when there is no maximum oscillation period.

A refractory period is required for rippling for cells of length greater than 2 or 3 nodes, and although there may exist a minimum response time of 
more than one time-step, it is an interesting result of our model that the minimum response period $T-R$ must be small compared to the refractory period. In particular, rippling occurs whenever the minimum oscillation time $t$ is greater than $\ell / v$ time-steps and the refractory period $R$ is at least two-thirds $t$. The first condition is required because if the minimum oscillation period $t$ is less than the period of time it takes a cell to travel one cell length, two cells or a cluster of cells will stimulate each other to oscillate in place. The second condition that the refractory period is at least two-thirds the minimum oscillation period indicates that the minimum response time of a cell can not be too long compared to the refractory period.

Experiments suggest that the minimum oscillation period of a cell in response to $\mathrm{C}$-factor is about 3 minutes [12]. According to our result that the minimum response time cannot be more than two-thirds the refractory period, we can predict the existence of a refractory period in myxobacteria cells, with a duration of 2-3 minutes.

The wavelength of the ripples depends on both the duration of the refractory period and the density of signaling cells. Figure 5 shows that the ripple wavelength increases with increasing refractory period (a) and decreases with increasing cell density (b). Notice that error bars that show standard deviations of the mean wavelength over five simulations increases with wavelength. A refractory period of 2-3 minutes yields a ripple wavelength of about $60 \mathrm{mi}-$ crometers (Figure 5a), which corresponds well to typical experimental ripple wavelengths [12]. The correspondence between refractory period and wavelength given in Figure 5 is a only rough estimate, however. We believe the reasons are that in these simulations the cell density is relatively low, which decreases the density of C-factor relative to experimental conditions, and cells are not very elongated, which increases the density of C-factor relative to experimental conditions.
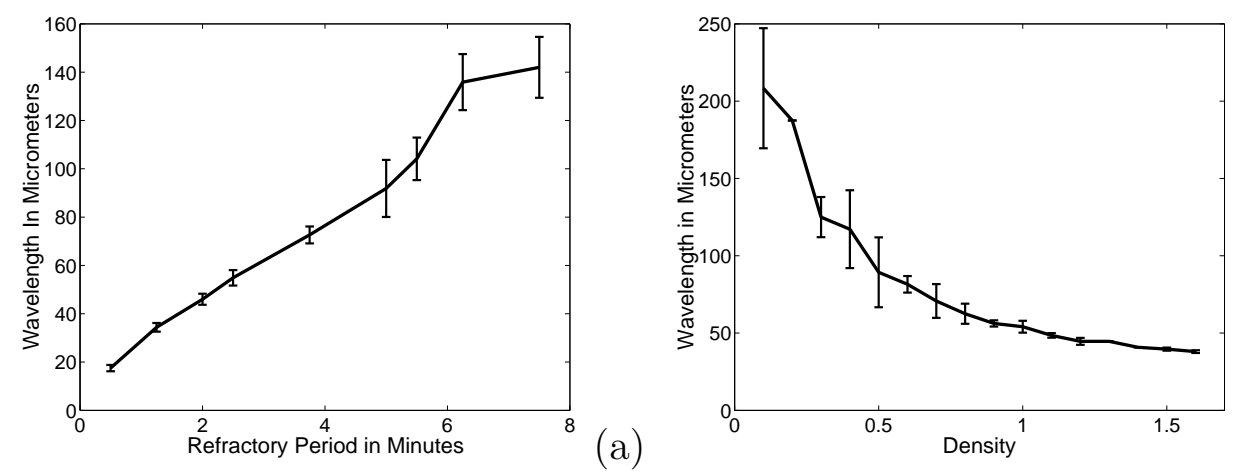

Fig. 5. a) Average wavelength in micrometers versus refractory period in minutes. Cell length $\ell=4, \delta=1$. The internal timer is adjusted so that the fraction of clock time spent in the refractory period is constant: $t=3 R / 2$ and $\tau=5 * R / 2$. b) Average wavelength in micrometers versus density (total cell area over total lattice area). Cell length $=4$ with an internal timer given by $R=8, t=12, \tau=20$. 
Note that in Figure 5a, the curve has a wavelength of approximately $20 \mathrm{mi}-$ crometers when the refractory period is less than 1 minute. Since cells have a length of 5 micrometers, this is the smallest wavelength that may be resolved as there is only one cell length between subsequent high density waves. At very high density, when the refractory period is 0, cells may be stimulated to reverse every timestep, so that there would be, theoretically, a wavelength of only 1 node. However, cells will be uniformly distributed in this case and there will be no well-defined high-density waves. In the simulations described in Figure 5b, density is increased while refractory and minimum oscillation periods have a constant value. The minimum possible wavelength in this case is limited by the minimum oscillation period. In particular, the minimum possible wavelength is twice the minimum distance traveled by a cell between reversals, which is twice the distance traveled during the minimum oscillation period, which is 30 micrometers in this example. Thus, even as density is increased very high, the curve must have a horizontal asymptote at wavelength $=30$ micrometers.

\subsection{Non-linear response of reversals to C-factor.}

Reversals depend on the number of collisions a cell encounters which depends on the density of C-factor. Thus the number of collisions required for a reversal, the quorum value $q$, should be a function of the density of C-signaling nodes. The density of C-signaling nodes is a function of both cell density and cell length since longer cells have a reduced C-signaling area to non-C-signaling area ratio. Thus, we describe optimal quorum values $q$ as a function of $\mathrm{C}$ signaling node density rather than cell density.

At a low density of C-signaling nodes, ripples form even when both $q$ and $p$ are 1 so that only 1 collision during the sensitive period is needed to trigger an reversal. When the density of C-signaling nodes is greater than or equal to 1 , however, the chances of collisions are so high in the initial homogeneous population that cells almost always reverse in the minimum number of timesteps and, with no differential behavior among cells, a rippling pattern fails to form. Ripples will form at arbitrarily large densities of cells and C-signaling nodes if the number of collisions needed to trigger a reversal is increased. When the number of collisions required for a reversal is greater than $3(q>3)$, ripples develop more quickly if the non-linear response to density $p$ is increased greater than 1 . A value of $p=3$ yields optimal rippling for all quorum values and densities, which is consistent with the results of Igoshin et al. [35] for their value of $p[35]$. 


\subsection{Ripple phase shift}

Counter-propagating ripples appear to pass through each other with no interference, which lead Sager and Kaiser to propose the hypothesis of precise reflection [12]. Indeed, tracking of right-propagating ripples and left-propagating ripples in Figure 8a, shows that the waves move continuously despite collisions and subsequent reflection. Inspection of the collision and subsequent reversal of two cells, however, shows there is a jump in phase equal to exactly one cell length if they reverse immediately upon colliding (see Figure 6a). This phase jump occurs because a cell reverses by changing its orientation rather than by turning: when a right-moving particle collides with a left-moving particle and reverses, it is exactly one cell length ahead of the left-moving cell that it replaces. When all of the particles within a ripple are in phase, as is often the case, this jump is also seen in the ripple waves as two waves interpenetrate. If the cells continue $p$ more steps before reversing (for example, if their clocks were almost near $\tau$ after the collision), then there would be a phase jump of $\ell-2 p$. If $2 p>\ell$, there will be a phase delay (see Figure $6 \mathrm{~b}$ ). In their continuous model, Igoshin et al. ([35], Figure 3b) also showed when ripples collide a small jump in phase reminiscent of a soliton jump.

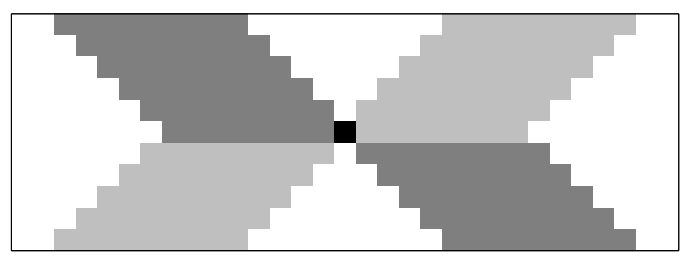

(a)

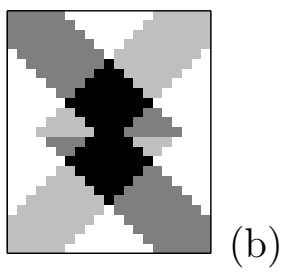

Fig. 6. Space-time plot of a wave inter-penetration. Time increases as the vertical axis descends. Right-directed particles are shown in dark gray, left-directed particles are shown in light gray. a) Phase jump of one cell length (9 units) as two cells collide and immediately reverse. b) Phase delay as two cells collide and travel 8 time-steps before reversing.

\subsection{Effect of dilution with non-signaling cells}

Sager and Kaiser [12] diluted C-signaling (wild-type) cells with non-signaling (csgA minus) cells that were able to respond to C-factor but not produce it themselves. When a collision occurs between a signaling and a non-signaling cell, the non-signaling cell perceives C-factor (and the collision), whereas the C-signaling cell does not receive C-factor and behaves as though it has not collided. The ripple wavelength increases with increasing dilution by non-Csignaling cells. Simulations of this experiment with and without an internal timer with a maximum oscillation period give very different results. Figure $7 \mathrm{a}$ shows that the dependence of wavelength on the fraction of wild type cells 
resembles the experimental curve only when there is no maximum oscillation period assumed in the model (compare with Figure 7.G in [12].) Thus our model predicts that rippling cells do not ripple with a maximum oscillation period. Notice that the range of wavelengths when there is no assumed maximum oscillation period is in good quantitative agreement with that of experiment (compare Figure 7a, solid line with Figure 7.G in [12].

Igoshin et al. [35] have previously reproduced the experimental relationship between wavelength and dilution with non-signaling cells (see [35] Supplemental materials, Figure 8) by adjusting their original internal timer. As the density of C-signal decreases, the phase velocity slows linearly and the maximum oscillation period of the internal timer increases continuously. Thus, the maximum oscillation period varies in their model. We assume a constant maximum oscillation period, which is either present or absent (longer than the simulation running time). Note that if the maximum oscillation period increases sufficiently with decreased density of C-factor so that a cell is always stimulated to turn before the internal timer would regulate a turn, then the addition of an internal timer is superfluous. In this case, the two models are similar.
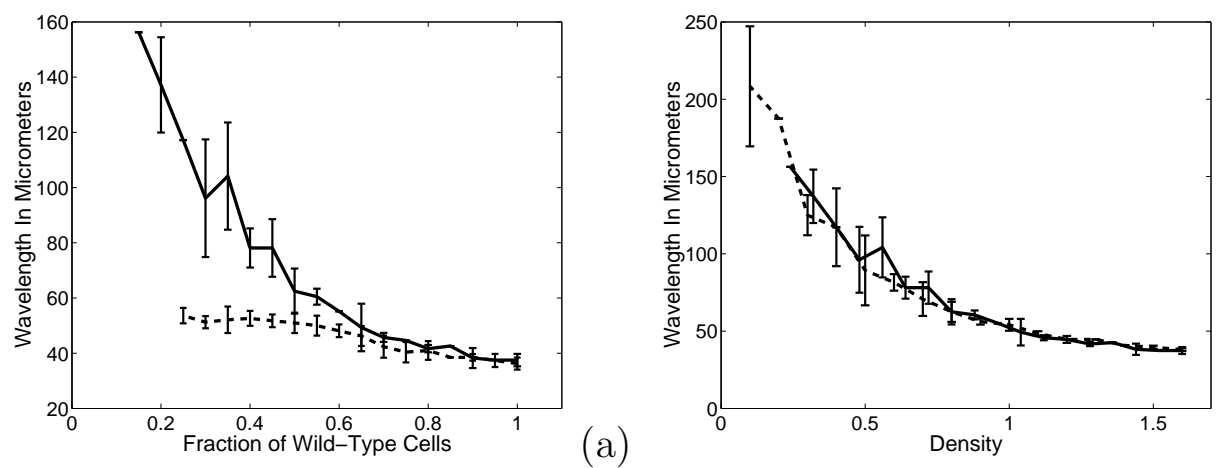

Fig. 7. a) Wavelength in micrometers versus the fraction of wild-type cells with (dotted line) and without (solid line) a maximum oscillation period. b) Wavelength in micrometers versus wild-type density with no $\operatorname{csg} \mathrm{A}$-minus cells (dotted line) and when the the density of csgA-minus cells is increased so that the total cell density remains 1.6 (solid line). Density is total cell area over total lattice area and there is no maximum oscillation period. For a) and b), cell length $=4, R=8, t=12$, $\tau=20$ (maximum oscillation period) or $\tau=2000$ (no maximum oscillation period).

Our simulations show ripple wavelength increases with increased dilution by non-signaling cells. Since wavelength also increases with decreasing density of signaling cells (Figure 5b), we ask if the mutant cells have any effect on the rippling pattern. Figure $7 \mathrm{~b}$ shows the wavelength dependence on the density of signaling cells when only signaling cells are present (dotted line) and for a mixed population of signaling cells of the same density with non-signaling cells added so that the total cell density is always 1.6 (solid line). Apparently, the decrease in C-factor explains the increase in wavelength. The non-signaling mutants do not affect the pattern at all. 
As density increases, wavelength decreases and the larger number of cells are distributed over a greater number of ripples. This result is further evidence of the the role $\mathrm{C}$-signaling plays as a density-sensing and density-modulating mechanism. To test this further, we ran a simulation for initial conditions in which a high density stripe stretches vertically down a lattice. As ripples formed and propagated, the cells were quickly distributed more evenly over the lattice (Figure 8b). The redistribution of cells occurs much faster than if cells moved randomly at each time-step (compare Figure 8, b and c). Thus, although there is no net transport of cells larger than one wavelength when cells are evenly distributed [12], there is net migration of cells away from high density regions.

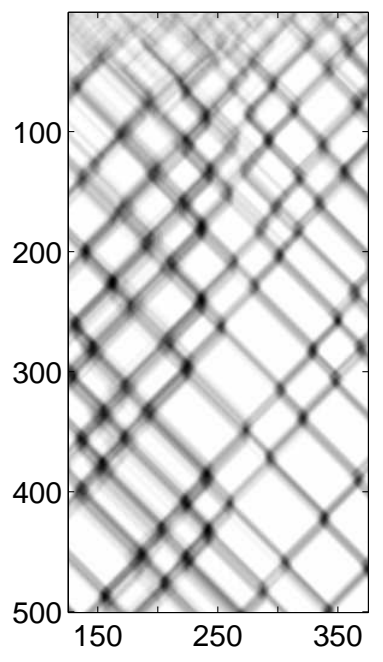

(a)
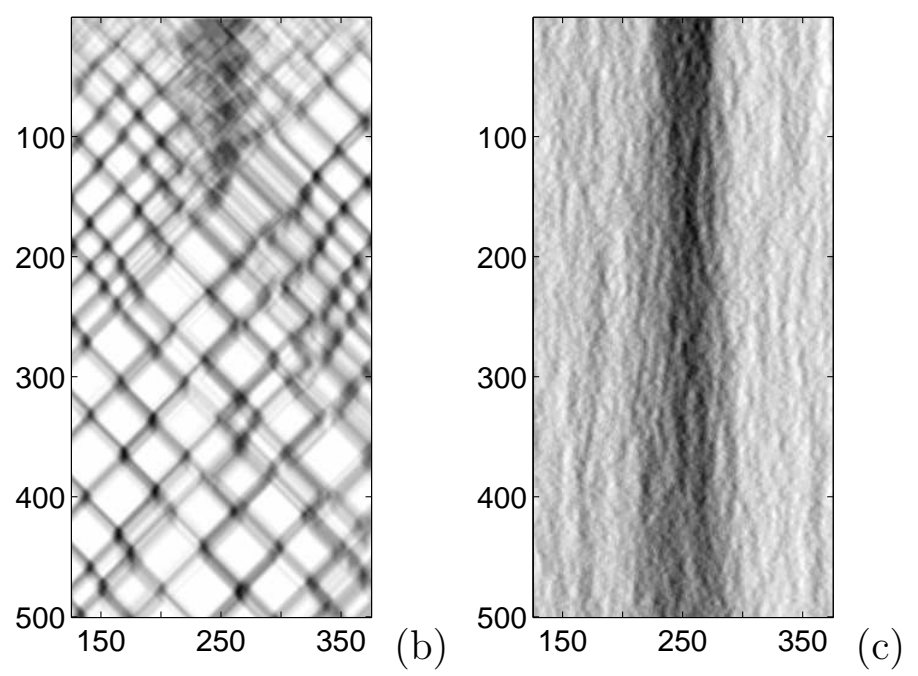

Fig. 8. Cell density over a subsection of the third row of a $5 \times 500$ lattice over the first 500 time-steps for different initial conditions. Time increases as the vertical axis descends. a) Cells are initially randomly distributed with density 3 . Cell length $=5$ nodes, $R=10$ nodes, $t=15$ nodes and $\tau=2000$. b) Same as in a), but with a central stripe of density 15 and width 50 initially added vertically down the lattice. c) Same as in b), but cells are assigned random orientations at every time-step.

\subsection{Discussion}

In our simulations, high-density waves of cells form from a homogeneous distribution of cells for a wide range of parameters and initial cell density. At low cell density when there is no assumed maximum oscillation period, the wavelength between ripples is large. The explanation for this is that a larger region of the lattice must be "swept" to collect an aggregate of cells with sufficiently high density to reverse another aggregate. In the extreme case where density is nearly zero, a single cell will keep traveling without ever encountering sufficient collisions, and the wavelength is infinite. Thus, the mechanism of rippling may be viewed as a mechanistic "sweeping" of an arbitrarily large 
area, in which cells modulate the area that they span between reversals so as to efficiently collect ripples of a minimum density.

At very high density, by the same argument, wavelength is small. The number of ripples per area increases so that the number of cells per ripple does not increase linearly with the increase in density, but less. Nevertheless, ripples formed from high density initial conditions do result in ripples which are wider and more dense than ripples formed from low density initial conditions. This may not be viewed as a limitation in design, but as the foundation of another possible role for rippling in myxobacteria: the even distribution of cells over an arbitrarily large area. When there are both high and low cell density regions (as in the simulation of Figure 8b), many high-density waves will form in the region of highest density, and fewer, lower density waves will form in the regions of lower density. At the interface between these regions, a high density wave encounters a lower density wave. The high density wave will reverse all the cells of the low density wave such that all the cells from the low density wave return toward the low density region. The cells of the low density wave, on the other hand, will not be able to reverse all the cells of the higher density wave. Rather, the lower density wave will only be able to reverse a proportional number of cells in the high density wave. The surplus cells in the high density wave will continue without reversing into the low density region. Thus, the rippling mechanism in a region of variable cell density creates a "pulse" of surplus cells within the high density region which is efficiently directed into lower density regions.

In summary, rippling is an efficient mechanism for both forming evenly spaced accumulations of high cell density, and evenly spaced accumulations of nearly equal cell density. In experiments, cells do not reflect by exactly 180 degrees. However, since most cells move roughly parallel to each other, models based on reflection are reasonable approximations. Modeling the experimental range of cell orientations would require a more sophisticated CA since LGCA require a regular lattice which does not permit many angles. In the aggregation section below we describe a model on a triangular lattice which could be adjusted to incorporate 120 degree reversals. Although a cell is ready to turn when the internal timer $\phi$ is greater than $\tau$, it may not be able to turn if the opposite channel is already occupied. This is another limitation of our LGCA model. We handle this situation by continuing to transport the cell in its direction of orientation at each time-step until the opposite channel is available. The effect of this delay is negligible, even at high densities within a ripple, when cells are so long that the probability of two cell centers occupying the same node is very small. 


\section{A preliminary model for aggregation}

Rippling is an intermediate, transient stage of fruiting body formation, which is not necessary for aggregation formation [32]. Figure 1 shows a field of aggregation centers surrounded by ripples. In this section we present a different LGCA model based on C-signaling alignment. This LGCA model reproduces the sequence and geometry of the non-rippling stages of fruiting body formation in detail, demonstrating how C-signaling-based alignment can account for these patterns with very few additional assumptions.

The non-rippling stages of fruiting body formation include alignment, streaming and aggregation. During alignment, cells form aligned patches from a random distribution. While streaming, cells form long chains which move cooperatively into aggregation center [30]. Aggregation is the phase in which cells form rounded collections that may either recede or mature into fruiting bodies. We model aggregation including only a simple local rule for C-signal-based alignment. The aggregates formed in our model are not species-specific and do not include local rules for rippling.

We use a hexagonal lattice since cell motion during aggregation is not onedimensional as in rippling. In this specialized LGCA model, identical rodshaped cells are all modeled as $3 \times \ell$ rectangles with $\mathrm{C}$-signal interaction neighborhoods as depicted in Figure 9. Cells move exactly one node per timestep in the direction of their orientation and there may only be one cell center per channel per node. In contrast to rippling, we find that the cell aspect ratio is an important parameter for streaming and aggregation, the simulations presented here all have a $1 \times 7$ aspect ratio for cells.
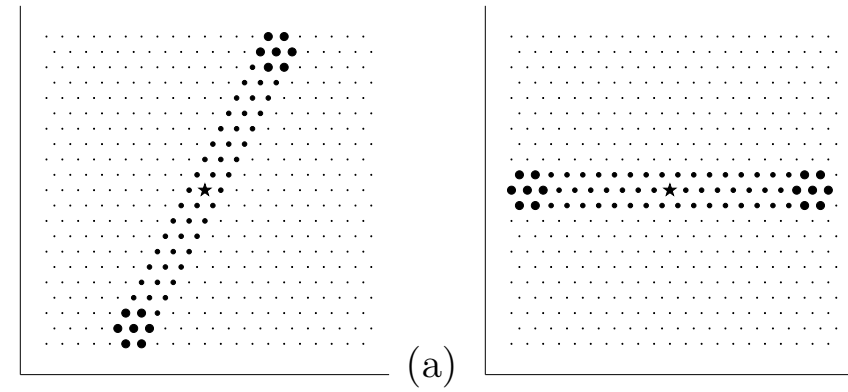

(b)

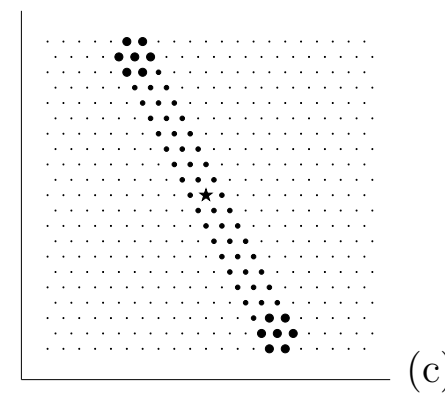

Fig. 9. The cell shapes of a) a cell oriented 60 or 240 degrees, b) a cell oriented 0 or 180 degrees and c) a cell oriented 120 or 300 degrees. All cells are $3 \times 21$ for a $1 \times 7$ aspect ratio. Each cell's "center of mass" is indicated by a star and the nodes of the interaction neighborhood where C-factor is exchanged are indicated by the larger black disks at the cell poles.

Myxobacteria align when they move. We choose an alignment based on Csignaling. We use a Monte Carlo process, in which cells turn 60 degrees clockwise, 60 degrees counter-clockwise or persist in their original direction with 
probability favoring directions that maximize overlap of C-signaling nodes. Interaction only occurs when the C-signaling nodes at the head of a cell overlap with the C-signaling nodes at the tail of another cell, and interaction occurs regardless of cell orientation. Head and tail C-signaling neighborhoods are shown in Figure 9.

We model C-signal alignment on a $256 \times 256$ lattice, in which our initial conditions are a random distribution of cells at high density. Within a few timesteps, cells form aligned patches (see Figure 10a). This reproduces the initial alignment stage of myxobacteria cells during fruiting body formation in which cells form an aligned patchwork [29]. Since there are only 6 directions permitted on the lattice, the aligned patchwork appears as a very regular triangular network. Cells are aligned both parallel and anti-parallel within each patch since the overlap of C-signaling nodes is maximized when cells are aligned regardless of their orientation. This geometry is significant because cells have naturally formed the aligned bi-directional arrays necessary for rippling. The local rules for rippling have been suppressed in this preliminary model, however, to evaluate the patterns formed by C-signaling-based alignment alone.

The homogeneous triangular network is not stable over time. As cells move, they turn and flow from one patch to another. Cells moving in a low density area are likely to turn into a higher density patch, so the network of cells condense into thick aggregates (Figure 10b). In experiments, a myxobacteria stream will often merge into an adjacent myxobacteria stream [41,42]. Figure $10 \mathrm{~b}$ shows that the cells move into the aggregates along streams directed into the aggregate, reproducing the streaming stage in which myxobacteria cells form long aggregates that move cooperatively [32].

The aggregates continue to condense while arranging and dividing into many small, circular orbits about 1.5 cell lengths (about 10-20 $\mu \mathrm{m}$ ) in diameter (Figure 10c). The aggregates often form in clusters of two or three closed orbits (Figure 10b), corresponding to fused aggregates (sporangioles). In Stigmatella erecta, several fruiting bodies may form in groups and fuse [28]. A magnified picture of the cell centers of a typical aggregate show that cells are arranged in dense, concentric layers tangent to a relatively low-density inner region (Figure 10d). Thus, they are geometrically equivalent to the basal region of aggregates in Myxococcus xanthus.

Cells in our simulation simultaneously move both clock-wise and counterclockwise around the aggregate, as they do in the fruiting bodies of Myxococcus xanthus [25]. The microscopic circular or elliptic orbits of Stigmatella spp. often disappear as cells spiral away from the aggregate [31]. Similarly most orbits in our simulation also eventually disappear as cells spiral away from the aggregate. Nevertheless, orbits typically survive for several hundred timesteps, which is about 5 to 10 complete revolutions for each cell. 
During myxobacteria aggregate formation, several aggregation centers will form and, inexplicably, one aggregation center will grow as an adjacent aggregation center disappears [32]. Our simulations offer a closer look at this process: a stream may form that connects two adjacent aggregation centers and, stochastically, cells stream from one aggregation center to another until the largest aggregation center absorbs the smaller one (Figure 10e).

Figure 10f shows several stable aggregates which have developed at 200 timesteps. Notice that the stable hollow aggregate has a much thicker annulus of cells than the non-stable orbits of Figure 10c, suggesting that only large hollow aggregates are stable. In our simulation at a threshold density within the aggregate, the hollow region of an aggregate will fill with cells such that cells are are arranged in six dense overlapping layers (Figure 10f). The third aggregate shown is a chain of cells which span the lattice and thus form an orbit due to the periodic boundary conditions of the lattice.

It has been proposed in [31] that circular motility at aggregation sites, trail following and local accumulation of slime account for fruiting body formation in Stigmatella spp. We hypothesize that once C-signaling has drawn cells into an aggregate, cell and slime cohesivity cause myxobacteria cells to round up into a mound while constant cell velocity pushes cells toward the surface of the mound, so that cells form a dense hemisphere of cells spiraling around a hollow center. In our model, a closed, circulating orbit of cells is the only stable configuration of a stationary aggregate since cells are constantly moving. At low and intermediate density, these orbits are hollow and cells are arranged tangentially within an annulus. At a threshold density, however, every channel of every node within the aggregate becomes occupied and there is no hollow center. We hypothesize that in a more advanced three-dimensional model, the addition of a local rule accounting cell and slime cohesivity will cause cells to round while maintaining the hollow center.

This model is preliminary because only C-signal based alignment is modeled and the aggregates formed are not species-specific. In this simulation, a 256x256 lattice size was chosen, which corresponds to an 80x80 $\mu \mathrm{m}$ region and about 10,000 cells for an averaged cell density of 10, much smaller compared to normal fruiting bodies that may be up to $1000 \mu \mathrm{m}$ in diameter. Aggregation in this model is described in more detail in [44].

This model can be adjusted to model rippling and aggregation concurrently by incorporating the local rules for rippling described in Section 3.2. Tracking of rippling cells in experiment (see Figure 6 in [12] and Figure 6 in [25]) suggest that reversals are about 150 degree changes in orientation rather than exactly 180 degrees, as we have assumed in the model for rippling of this paper. On a triangular lattice, a reversal of 120 degrees would be an equally good approximation of the 150 degree rotation. C-signaling based alignment 

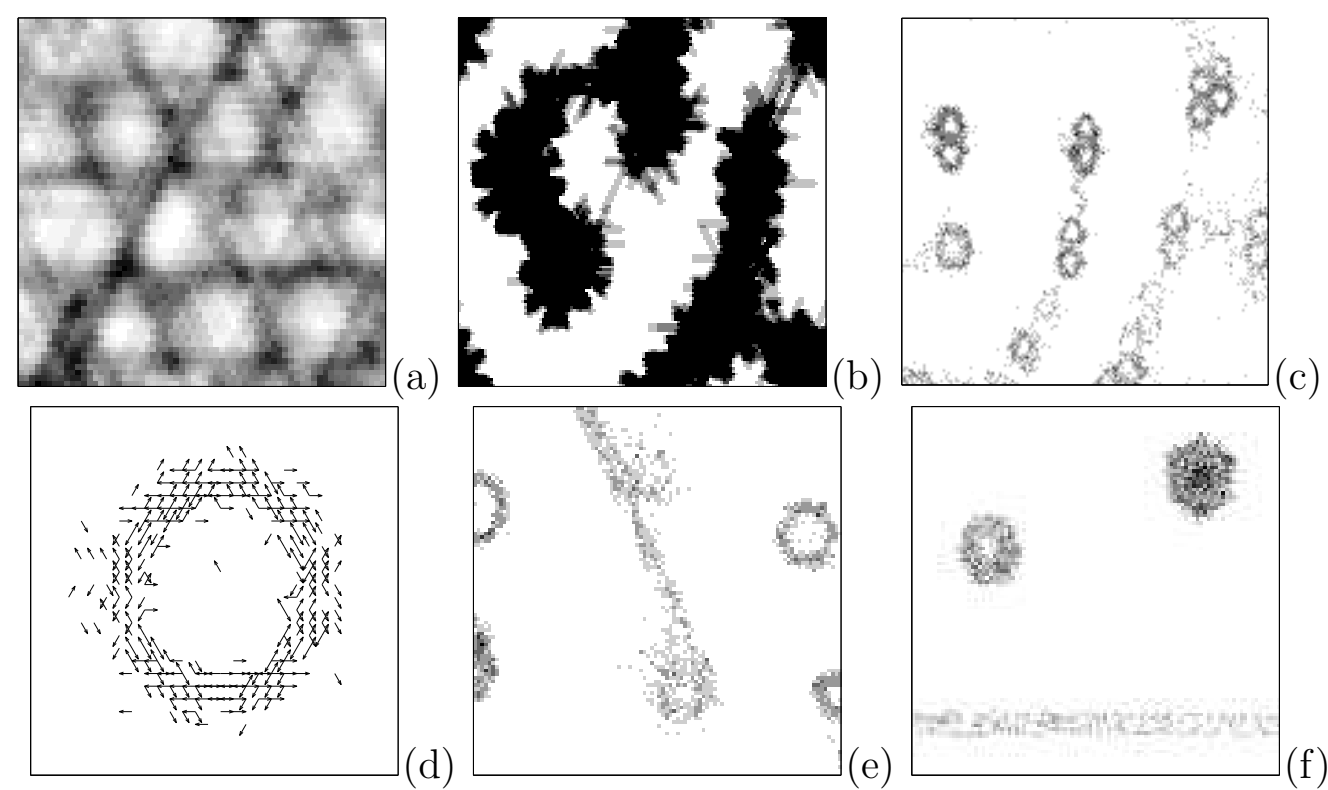

Fig. 10. Cell density development by C-signal alignment on a $256 \times 256$ lattice. Initial cell density is 10 . Cell density a) at 25 time-steps $(64 \times 64$ lattice sub-section $)$ and b) at 100 time-steps $(128 \times 128$ lattice sub-section $)$. Cell centers c) at 200 time-steps $(150 \times 150$ lattice sub-section $)$, d) of a $24 \times 24$ lattice sub-section with arrows indicating direction (450 time-steps), e) at 450 time-steps $(100 \times 100$ lattice subsection) and f) at 2000 time-steps $(100 \times 100$ lattice sub-section $)$.

combined with local rules for rippling would ensure that the majority of cells still remain parallel. Nevertheless, a regular shift in orientation by 120 or 180 degrees may have an interesting effect on the final distribution of cells and, subsequently, fruiting bodies.

Fruiting bodies among different myxobacteria species are very diverse (see Figure 3 in [43]). For example, while Myxococcus fruiting bodies are a relatively simple, single mound of cells, other species form clusters of mounds called sporangioles that are raised on a stalk. The interaction of adventurous verses social motility may account for these different morphologies [32]. During stalk formation of Stigmatella spp., cells are arranged perpendicularly to the mound as the fruiting body is lifted $[31,33]$. Also, fruiting body stalks may be composed of a larger, second cell-type [31]. Thus, once the stages of fruiting body formation have been modeled in general, it would be interesting to determine which parameters need to be varied to model the fruiting bodies of different species. 


\section{Summary}

In this paper, we present a new LGCA approach for modeling cells which is computationally efficient yet approximates continuum dynamics more closely than assuming point-like cells. As an example of this new approach, we present a model for myxobacteria rippling based on the hypothesis of precise reflection. The results of our model show that rippling is stable for a wide range of parameters, C-signaling plays an important role in modulating cell density during rippling, and non-C-signaling cells have no effect on the rippling pattern when mixed with wild-type cells. Further, by comparing model results with that of experiment, we can conclude reversals during rippling would not be regulated by a built-in maximum oscillation period. We also present a second LGCA model based on C-signal alignment which reproduces the sequence and geometry of the non-rippling stages of fruiting body formation in detail, showing that a simple local rule based on C-signaling can account for many experimental observations.

\section{Acknowledgments}

We would like to thank Dale Kaiser, Frithjof Lutscher and Stan Marée for very helpful discussions. MSA is partially supported by grant NSF IBN-0083653. YJ is supported by DOE under contract W-7405-ENG-36. MAK is supported by the Center for Applied Mathematics and the Interdisciplinary Center for the Study of Biocomplexity, University of Notre Dame, and by DOE under contract W-7405-ENG-36.

\section{References}

[1] D. Kaiser and L. Kroos, in: M. Dworkin and D. Kaiser (Eds), Myxobacteria II, Am. Soc. Microbiol., Washington DC, 1993.

[2] Y. Jiang, H. Levine, and J.A. Glazier, Biophys. J. 75 (1998) 2615.

[3] A.F.M. Marée, Ph.D. Thesis., Utrecht University, 2000.

[4] A.F.M. Marée and P. Hogeweg, Proc. Natl. Acad. Sci. USA 98 (2001) 3879.

[5] M. Dworkin, Microbiol. Rev. 60 (1996) 70.

[6] R.P. Buchard, Ann. Rev. Microbiol. 35 (1981) 497.

[7] C. Wolgemuth, E. Hoiczyk, D. Kaiser and G. Oster, Curr. Biol. 12 (2002) 369.

[8] J. Hodgkin and D. Kaiser, Mol. Gen. Genet. 171 (1979) 167; ibid. 177.

[9] H. Reichenbach, Ber. Dtsch. Bot. Ges. 78 (1965) 102. 
[10] L.J. Shimkets and D. Kaiser, J. Bacteriol. 152 (1982) 451.

[11] R. Welch and D. Kaiser, Proc. Natl. Acad. Sci. USA 98:26 (2001) 14907.

[12] B. Sager and D. Kaiser, Genes Dev. 8 (1994) 2793.

[13] R.P. Buchard, in: E. Rosenburg (Ed), Myxobacteria, Springer-Verlag, New York, 1984.

[14] T.M.A. Gronewold and D. Kaiser, Mol. Microbiol. 40 (2001) 744.

[15] S.K. Kim and D. Kaiser, Cell 61 (1990) 19.

[16] S.K. Kim and D. Kaiser Genes Dev. 4 (1990) 896.

[17] S.K. Kim and D. Kaiser, Science 249 (1990) 926.

[18] L. Kroos, P. Hartzell, K. Stephens and D. Kaiser, Genes Dev. 2 (1988) 1677.

[19] D. Hagen, A. Bretscher, and D. Kaiser, Dev. Biol. 64 (1978) 284.

[20] L.J. Shimkets, R.E. Gill and D. Kaiser, Proc. Natl. Acad. Sci. USA 80 (1983) 1406.

[21] L.J. Shimkets and H. Rafiee, J. Bacteriol. 172 (1990) 5299.

[22] S.K. Kim and D. Kaiser, J. Bacteriol. 173 (1991) 1722.

[23] S. Li, B. Lee and L.J. Shimkets, Genes Dev. 6 (1992) 401.

[24] S.K. Kim, D. Kaiser, and A. Kuspa, Ann. Rev. Microbiol. 46 (1992) 117.

[25] B. Sager and D. Kaiser, Proc. Natl. Acad. Sci. USA 90 (1993) 3690.

[26] B. Julien, D. Kaiser and A. Garza, Proc. Natl. Acad. Sci. USA 97 (2000) 9098.

[27] T. Kruse, S. Lobedanz, N.M. Berthelsen, and L. Søogaard-Andersen, Mol. Microbiol. 40 (2001) 156.

[28] H. Reichenbach, in: M. Dworkin and D. Kaiser (Eds), Myxobacteria II, Am. Soc. Microbiol., Washington DC, 1993.

[29] D. Kaiser, in: R. England, G. Hobbs, N. Bainton and D. McL.Roberts, Microbial Signaling and Communication, Cambridge University Press, UK, 1999.

[30] L. Jelsbak and L. Søgaard-Andersen, Curr. Opin. Microbiol. 3 (2000) 637.

[31] D. White, in: M. Dworkin and D. Kaiser (Eds), Myxobacteria II, Am. Soc. Microbiol., Washington DC, 1993.

[32] D. Kaiser (private communication).

[33] G.M. Vasquez, F. Qualls, and D. White, J. Bacteriol. 163 (1985) 515.

[34] U. Börner, A. Deutsch, H. Reichenbach, and M. Bär, Phys. Rev. Lett. 89 (2002) 078101. 
[35] O. Igoshin, A. Mogilner, D. Welch, D. Kaiser, and G. Oster, Proc. Natl. Acad. Sci. USA 98 (2001) 14913.

[36] F. Lutscher and A. Stevens, J. Nonlinear Sci. 12 (2002) 619.

[37] L. Jelsbak and L. Søgaard-Andersen, Proc. Natl. Acad. Sci. USA 96 (1999) 5031.

[38] W. Shi, F. Ngok and D. Zusman, Proc. Natl. Acad. Sci. USA 93 (1996) 4142.

[39] A. Goldbeter, Biochemical oscillations and cellular rhythms, Cambridge University Press, Cambridge, 1996.

[40] S. Dormann, Dissertation, Universität Osnabrück, 2000.

[41] A. Stevens, SIAM J. of Appl. Math. 61 (2000) 172.

[42] M. McBride, P. Hartzell and D. Zusman in: M. Dworkin and D. Kaiser (Eds), Myxobacteria II, Am. Soc. Microbiol., Washington DC, 1993.

[43] D.H. Pfister, in: M. Dworkin and D. Kaiser (Eds), Myxobacteria II, Am. Soc. Microbiol., Washington DC, 1993.

[44] M.S. Alber, Y. Jiang, and M.A. Kiskowski (submitted). 